\title{
Avenir des nouveaux concepts des calculs dosimétriques basés sur les méthodes de Monte Carlo
}

\author{
L. MAKOVICKA ${ }^{1}$, A. VASSEUR ${ }^{1,2}$, M. SAUGET ${ }^{1,2}$, E. MARTIN $^{1}$, \\ R. GSCHWIND ${ }^{1}$, J. HENRIET ${ }^{1}$, M. SALOMON ${ }^{2}$
}

(Manuscrit reçu le 30 septembre 2008, accepté le 2 décembre 2008)

RÉSUMÉ Les codes de calculs Monte Carlo, précis mais lents, sont devenus des outils incontournables dans la grande majorité des spécialités liées à la radiophysique, la radioprotection et la dosimétrie. Une réflexion mérite d'être menée sur l'opportunité d'implémenter d'autres solutions informatiques qui ne seraient pas seulement basées sur l'unique puissance informatique ou sur un «biaisage » permettant une relative accélération de ces calculs dans les cas des rayonnements photoniques, mais sur des méthodes plus judicieuses (RNA-réseaux de neurones artificiels, RàPC raisonnement à partir de cas ou exploitation d'autres techniques informatiques), déjà utilisées avec succès depuis longtemps dans certaines applications scientifiques ou industrielles dans les domaines souvent éloignés de la radioprotection ou de la dosimétrie médicale.

ABSTRACT The future of new calculation concepts in dosimetry based on the Monte Carlo methods.

Monte Carlo codes, precise but slow, are very important tools in the vast majority of specialities connected to Radiation Physics, Radiation Protection and Dosimetry. A discussion about some other computing solutions is carried out; solutions not only based on the enhancement of computer power, or on the "biaising" used for relative acceleration of these codes (in the case of photons), but on more efficient methods (ANN - artificial neural network, CBR - case-based reasoning — or other computer science techniques) already and successfully used for a long time in other scientific or industrial applications and not only Radiation Protection or Medical Dosimetry.

Keywords: Monte Carlo / artificial neural network / case-based reasoning / radiotherapy / radiation protection

\section{Introduction}

La meilleure représentation de la réalité physique des interactions des rayonnements ionisants avec la matière nous est apportée en règle générale par les codes basés sur les méthodes Monte Carlo, connues non seulement pour leur

1 Université de Franche-Comté, Équipe IRMA/ENISYS/FEMTO-ST, UMR6174 CNRS, BP 71427, 25211 Montbéliard Cedex, France.

2 Université de Franche-Comté, Équipe AND/LIFC, rue Engel Gros, 90000 Belfort, France. 
souplesse dans la prise en compte et l'implémentation de paramètres nouveaux, mais aussi pour leur lenteur, devenue probablement le principal handicap pour leur meilleure exploitation et leur développement dans les secteurs d'application où la rapidité est aussi importante que la précision (applications médicales, reconstitution d'accidents radiologiques...).

Les différentes solutions proposées actuellement pour accélérer les calculs Monte Carlo sont essentiellement basées sur le «biaisage» des codes et sur l'exploitation maximale de la puissance informatique. Cette voie dont la faisabilité a été démontrée dans le cadre de certains projets (Kafrouni et al., 2008), y compris européens (MAESTRO), risque néanmoins d'être une solution lourde, coûteuse et donnant peu d'autonomie à l'utilisateur. Le biaisage, même validé dans des cas précis, pourrait être un frein à l'évolution des codes MC pour la prise en compte de nouveaux phénomènes physiques, biologiques ou chimiques.

L'équipe IRMA s'oriente depuis quelques années vers l'utilisation de concepts informatiques autres, utilisés dans différentes applications scientifiques ou techniques. Les représentants les plus connus sont les RNA (réseaux neuronaux artificiels). Les études de faisabilité, assez longues et laborieuses, ont été effectuées en grande partie grâce à la collaboration étroite avec l'équipe d'informatique AND/LIFC dans le cadre des stages de fin d'études ou de thèses (Mathieu et al., 2003, 2004a, 2004b, 2005 ; Bahi et al., 2006a, 2006b ; Sauget et al., 2005 ; Sauget, 2007, Vasseur et al., 2006, 2008 ; Bopp et al., 2009). Ces travaux montrent que les techniques d'apprentissage peuvent trouver leur place aussi bien en radiothérapie qu'en radioprotection.

Après avoir présenté les contraintes et les enjeux de la radiothérapie externe dans une deuxième partie ainsi que les principes des RNA, du RàPC, et les intérêts qu'ils présentent pour aider à répondre à cette problématique, nous étudierons les résultats obtenus avec l'utilisation des RNA pour la radiothérapie externe.

\section{Radiothérapie externe, radioprotection et techniques informatiques innovantes}

\subsection{Problématique de la radiothérapie externe}

Le but de la radiothérapie externe est le traitement des tumeurs tout en épargnant le plus possible les tissus sains avoisinants. Afin de maitriser et d'optimiser la distribution des doses délivrées au patient, une planification dosimétrique doit être effectuée au préalable. Pour déterminer la répartition des doses, la routine thérapeutique est confrontée à la recherche du meilleur compromis entre la précision et la rapidité d'obtention de cette information. Les techniques basées sur les 
méthodes analytiques ou sur des bases de données préalablement établies ont le mérite d'être rapides, mais peu souples. Leur précision n'est assurée que dans les cas directement prévus. Dans cette optique, l'utilisation des méthodes telles que celles de Monte-Carlo apporte plus de précision. Cependant, malgré tous les efforts fournis aussi bien sur le plan de l'optimisation des méthodes que sur le plan de l'évolution informatique (puissance de calcul brute des machines), elles se heurtent au problème du temps de calcul. Nous avons envisagé, en rassemblant les avantages de ces différentes approches, d'utiliser et de développer des techniques basées sur les principes des réseaux d'apprentissage. Les domaines de la radiothérapie et de la radioprotection peuvent être considérés, malgré leur relative complexité, comme bien adaptés à ce type d'exercice car les conditions géométriques, la composition matérielle des tissus irradiés, les éléments concernés ou les différentes énergies des sources restent relativement limités et se prêtent $a$ priori à l'exploitation de ces «interpolations intelligentes». À ce titre, l'introduction des méthodes RàPC pourrait être également envisagée, plus particulièrement pour les validations des traitements planifiés ou des reconstitutions de fantômes personnalisés.

\subsection{Réseaux neuronaux (RNA)}

Un réseau de neurones artificiels (Artificial Neural Network) est un modèle de calcul dont la conception est très schématiquement inspirée du fonctionnement des neurones biologiques (Figs. 1a et 1b). Le principe de ce modèle est connu depuis une bonne soixantaine d'années et la base de l'analogie avec la biologie est notamment discutée dans la publication Pitts et Culloch (1943).

Les réseaux de neurones sont généralement optimisés par des méthodes d'apprentissage de type statistique, si bien qu'ils sont placés d'une part dans la famille des applications statistiques, qu'ils enrichissent avec un ensemble de paradigmes permettant de générer de vastes espaces fonctionnels, souples et partiellement structurés, et d'autre part dans la famille des méthodes de l'intelligence artificielle qu'ils enrichissent en permettant de prendre des décisions s'appuyant davantage sur la perception que sur le raisonnement logique formel.

Les réseaux d'apprentissage, les RNA en particulier, semblent donc être une troisième voie combinant l'utilisation de données dosimétriques existantes obtenues aussi bien par la voie expérimentale que par simulations numériques (Monte Carlo ou analytiques).

De manière générale les RNA permettent d'inférer, à partir d'échantillons, un signal dont les valeurs peuvent être réelles, vectorielles ou discrètes. Dans les deux cas, il s'agit de construire un outil permettant de réaliser une correspondance entre des entrées et des sorties. Ainsi les RNA, souvent utilisés pour des problèmes de 
a)

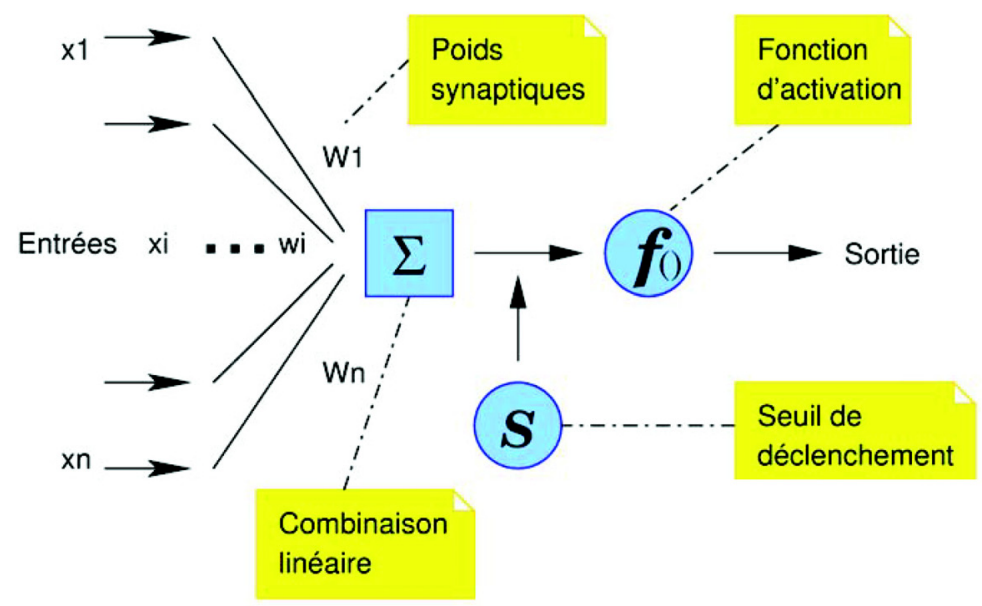

b)

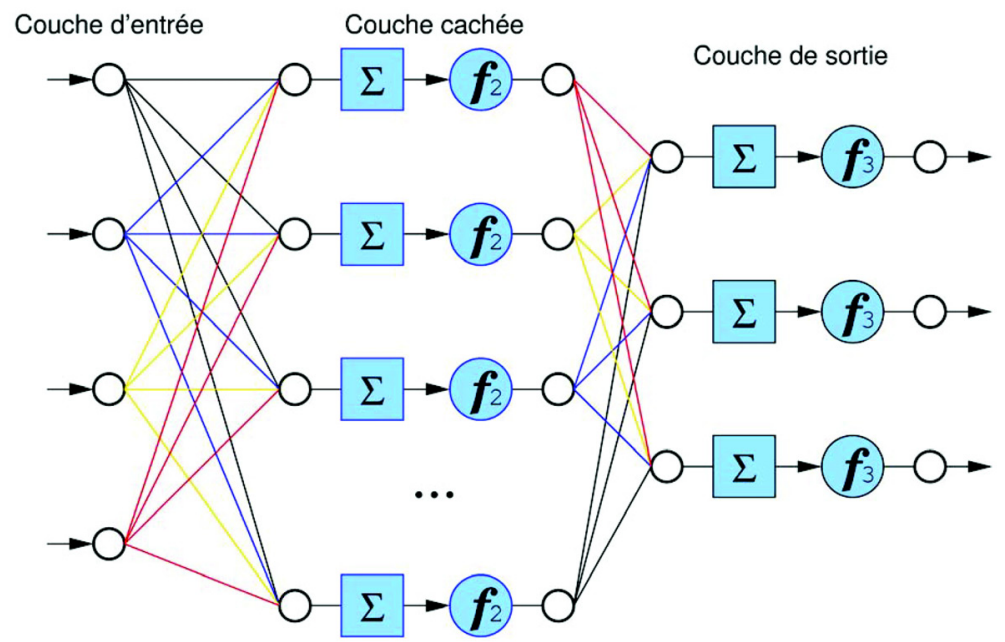

Figure 1 - (a) Principe d'un neurone artificiel; (b) exemple d'un réseau de neurones artificiels. (a) Principle of the artificial neuron; (b) ANN example.

classification, peuvent aussi être employés pour des problèmes d'approximation de fonction. On parle parfois de régression ou de modélisation «boîte noire » (pour souligner que le phénomène modélisé n'est pas explicité). Ainsi la sortie représente la valeur d'une fonction continue ; il s'agira dans notre cas de la dose 


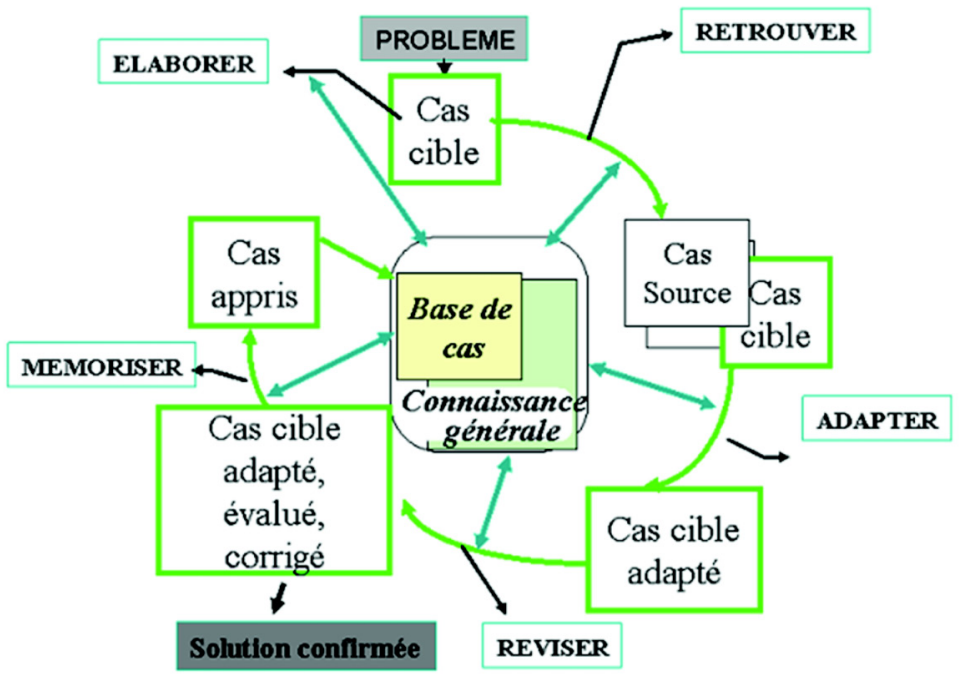

Figure 2-Cycle de fonctionnement du RàPC.

CBR methodology.

absorbée en fonction des paramètres liés à la géométrie et à la nature de la zone à irradier ainsi que les paramètres et les conditions d'irradiation.

\subsection{Raisonnement à partir de cas (RàPC)}

Le raisonnement à partir de cas (RàPC) est un outil d'apprentissage et de résolution de problèmes basé sur la réutilisation de solutions à des problèmes similaires appelés cas et stockés dans une base de cas. La représentation d'un cas peut varier suivant l'utilisation qu'il est faite de cet outil : diagnostic, planification, aide à la décision, conception, etc. Tout cas est composé d'une partie décrivant le problème, et de sa solution (Kolodner, 1993). La figure 2 représente le principe général de fonctionnement d'un RàPC. Nous avons prévu d'utiliser cette technique pour créer, reconstruire ou compléter des fantômes personnalisés. Cette approche peut être assez intéressante pour le domaine de la radiothérapie (cas de personnes ne pouvant pas soit subir tous les examens nécessaires, soit présentant des diagnostics incomplets, voire déformés, comme c'est parfois le cas de patients avec des implants artificiels - Gschwind et al., 2008) et surtout pour certains cas spécifiques de la reconstruction d'accidents radiologiques.

Tous les fantômes connus accompagnés de leurs caractéristiques sont stockés dans une base de cas sous la forme de «cas source». En saisissant les caractéristiques mesurables et connues d'un individu, il est possible de créer un 
«cas cible » (étape «Elaborer », Fig. 2). Grâce à une recherche en base de cas (étape «Retrouver»), les cas sources dont les caractéristiques sont les plus proches du cas cible sont présentés au praticien. Le fantôme du cas source le plus pertinent, choisi par l'expert, est ensuite adapté au cas cible (étapes « Adapter » et « Réviser»). Le fantôme révisé peut donc être utilisé pour établir un bilan dosimétrique de la victime. Ce nouveau fantôme est ensuite stocké avec ses caractéristiques dans la base de cas (étape « Mémoriser»). Ainsi le RàPC assure un retour d'expérience et une capitalisation des fantômes voxélisés connus, enrichissant la base de cas.

Le calcul de similarité, testé par notre équipe (Bopp et al., 2009), est accompagné d'un indice de confiance donnant une importance relative à chacune des mesures faites. En effet cette technique doit permettre de retrouver rapidement le ou les fantômes les plus semblables en fonction des informations mesurées avec l'indice de confiance qui est quant à lui, fonction des informations non mesurées. Même si toutes les caractéristiques ne sont pas saisies (car non mesurables par exemple), on peut retrouver un ensemble de fantômes similaires. Ce type de technique peut être associé à une utilisation simultanée des RNA.

\section{Principe d'utilisation des RNA et résultats obtenus}

L'idée initiale de combiner les méthodes de Monte Carlo avec les RNA pour établir un bilan dosimétrique rapide a été présentée par notre équipe en 2003 (Mathieu et al., 2003). Cette technique (Fig. 3) nécessite la mise en place d'une phase préparatoire assez complexe, laborieuse et importante en termes d'investissement humain dans son implémentation. Cette relative difficulté est liée essentiellement à la spécificité (besoin de compétences très pluridisciplinaires), à la complexité du domaine et surtout au manque significatif de références bibliographiques sur ce type précis d'application. Elle se montre par contre peu coûteuse sur le plan matériel, simple d'utilisation, autonome et surtout elle apporte un gain considérable en temps de calcul, aux méthodes Monte Carlo « classiques» ou « accélérées » par une puissance informatique conséquente.

Les calculs se décomposent en deux phases (Fig. 3) : une phase dite d'Apprentissage et une phase dite d'Évaluation. La phase d'apprentissage (encore appelée Entraînement du Réseau) permet la construction du réseau proprement dite. Si elle peut-être très coûteuse en temps de calcul (à l'instar d'un calcul MC par exemple), elle a l' avantage de pouvoir être faite au préalable. À titre d'exemple, l'apprentissage peut-être comparé aux calculs et à l'intégration des données dosimétriques de base faits initialement par les programmeurs de système de planification de traitements, lorsque leur logiciel est installé dans un nouveau service de radiothérapie. En résumé, ces calculs d'apprentissages sont accomplis « offline » et n'ont pas à être refaits pendant la phase de calcul de l'utilisateur (Évaluation). 


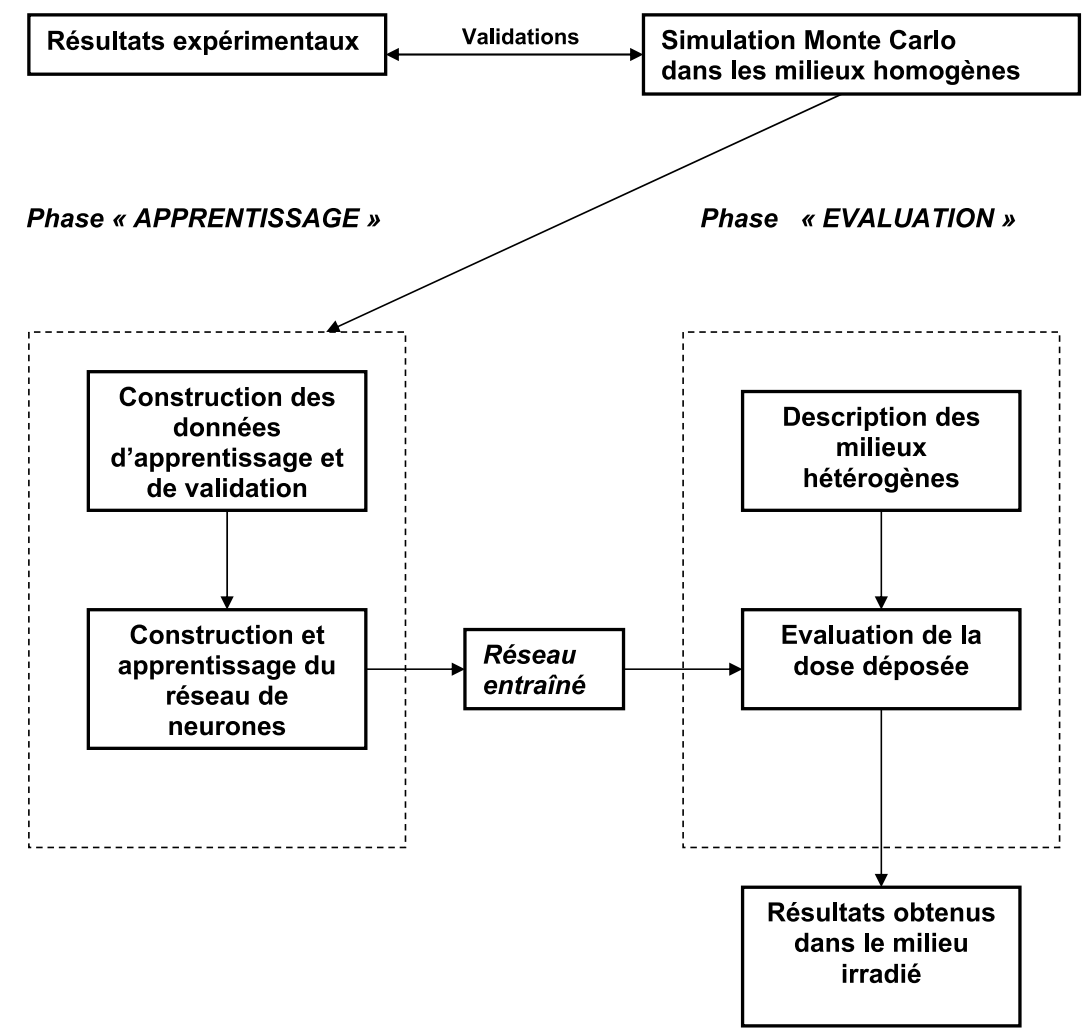

Figure 3 - Principe d'utilisation combinée des RNA - Monte Carlo pour le calcul des doses.

Principle of use of ANNs and MC dose calculations in combination.

Une fois le réseau entraîné au préalable, tout l'intérêt des RNA réside dans le fait que les calculs utilisateurs ne consomment que très peu de temps machine. En effet, si l'obtention d'un profil de dose à une seule profondeur (2D) par méthode MC (code du type EGS) requiert un temps de calcul de l'ordre de plusieurs heures, voire de plusieurs jours selon les moyens informatiques mis à disposition et la précision demandée, les réseaux de neurones artificiels, quant à eux, fournissent une réponse très inférieure à la minute, en $3 \mathrm{D}$, sur une géométrie identique et en utilisant un simple PC d'entrée de gamme (Mathieu et al., 2005).

Les résultats obtenus dans toutes les étapes mises en place jusqu'à présent, sans aucune introduction de paramètres de correction (divergences des faisceaux, effets d'interfaces, ...) se sont montrés très encourageants aussi bien dans les milieux homogènes (Fig. 4) que dans les milieux hétérogènes à géométrie relativement 


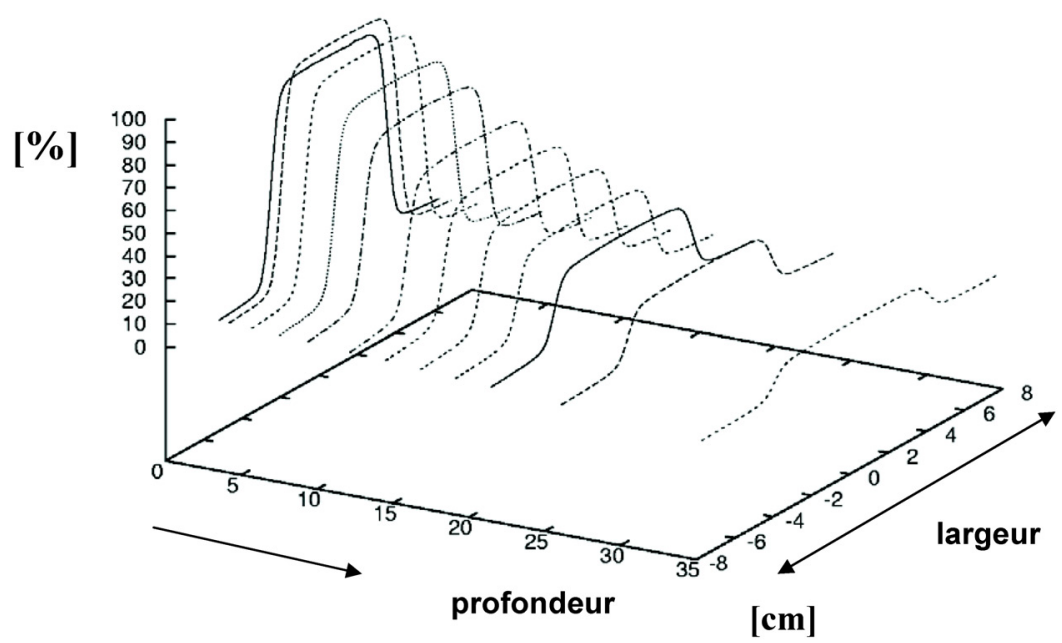

Figure 4 - Calculs, par un RNA, des rendements en profondeur dans l'eau, obtenus pour un faisceau de photons de SATURNE $43\left(4 \times 4 \mathrm{~cm}^{2}, 6 \mathrm{MeV}\right)$.

Relative depth doses calculated inside water using an ANN and obtained for photons of SATURNE $43\left(4 \times 4 \mathrm{~cm}^{2}, 6 \mathrm{MeV}\right)$.
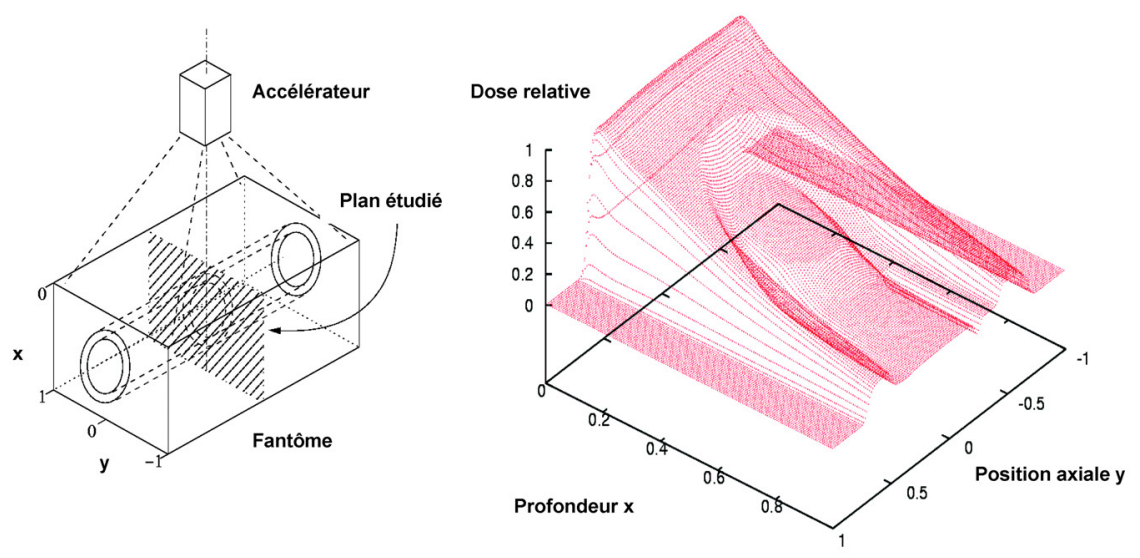

Figure 5 - Calcul des profils de doses dans les milieux hétérogènes à géométrie complexe.

Dose profiles calculated using $A N N$, inside heterogeneous materials of complex geometry.

complexe, un cylindre creux en titane plongé dans une cuve d'eau (profondeur $60 \mathrm{~cm}$, largeur $30 \mathrm{~cm}$, faisceau $10 \times 10 \mathrm{~cm}^{2}-$ Fig. 5). La Figure 4 montre un exemple de calcul de profils de doses relatives en profondeurs dans l'eau obtenus pour un faisceau de photons de SATURNE $43\left(4 \times 4 \mathrm{~cm}^{2}, 6 \mathrm{MeV}\right)$ réalisé par un 
a)

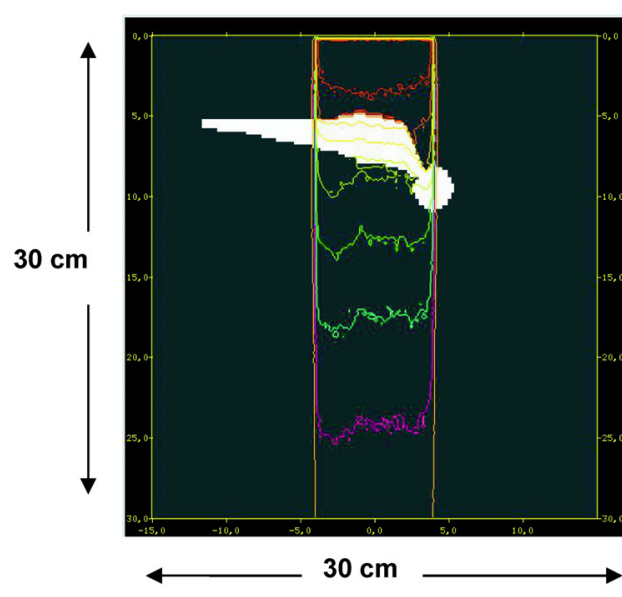

c)

b)

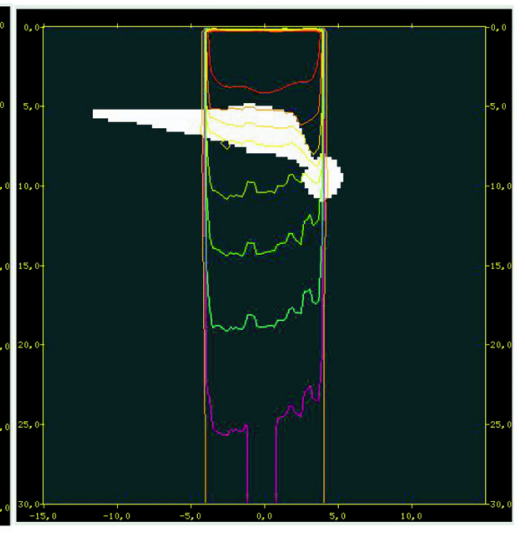

d)

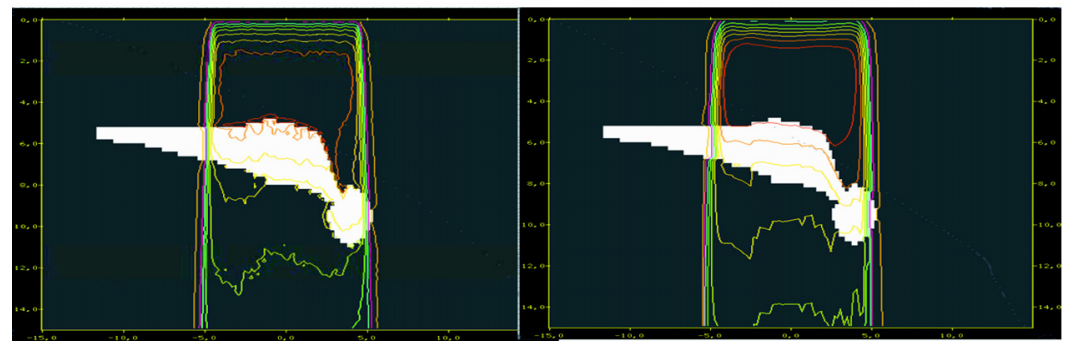

Figure 6 - Calcul de doses autour d'une prothèse de hanche plongée dans un fantôme d'eau : (a) MonteCarlo, faisceau parallèle, (b) RNA, faisceau parallèle, (c) Monte-Carlo, prise en compte de la divergence du faisceau, (d) RNA, prise en compte de la divergence du faisceau.

Dose calculations involving a hip prosthesis inside a water phantom: (a) Monte Carlo, parallel beam, (b) ANN, parallel beam, (c) Monte Carlo, conic beam, (d) ANN, conic beam.

réseau neuronal. Les figures 6 montrent la distribution de doses autour d'une prothèse de hanche plongée dans un fantôme d'eau.

Les principales différences obtenues entre le résultat de calcul NEURAD (Bahi et al., 2006a, 2006b) et le résultat de calcul MC, tiennent aux deux points suivants :

a. les calculs MC présentent un bruit statistique que l'on ne retrouve pas dans les calculs RNA. En effet, ces derniers fonctionnant à l'instar d'un interpolateur, un lissage (au sens mathématique du terme) est de facto obtenu (Mathieu et al., 2005) ; 
b. l'impact dosimétrique en sortie de prothèse n'est pas pris en compte. En effet, la rupture franche entre deux milieux de densités très différentes (en l'occurrence l'alliage de titane de la prothèse et l'eau) provoque localement un artefact de dose dû à un surplus relatif de diffusion d'électrons secondaires côté eau et à un déficit relatif de diffusion d'électrons secondaires côté prothèse. Ce phénomène a été démontré en détail par Buffard (Buffard et al., 2006).

Malgré tout, même dans ce cas de figure défavorable, les différences $\mathrm{MC} / \mathrm{NEURAD}$ restent globalement inférieures à $5 \%$ (seuil fixé en radiothérapie).

En ce qui concerne les temps de calculs pour l'obtention des résultats de la prothèse de hanche (Figs. 6), les calculs MC (Figs. 6a et 6c) ont nécessité plusieurs jours de calculs sur une station de calcul bi-Xeon $3 \mathrm{GHz}$, tandis que le calcul NEURAD fut de moins d'une minute sur un PC monoprocesseur P4 à $3 \mathrm{GHz}$.

L'ensemble des résultats obtenus prouvent que les réseaux de neurones peuvent apprendre et leur utilisation généralisée à partir de simples données d'entrée et de sortie (Vasseur et al., 2008). Il est ainsi possible de développer un modèle sans disposer de formule exprimant le phénomène modélisé, les seuls paramètres requis étant ceux directement liés à l'irradiation.

Mis à part le fait que les RNA peuvent fournir au final des résultats de calculs à la fois rapides et précis, leur principale force vient du fait que les moyens informatiques requis sont tout à fait négligeables devant ceux que nécessitent souvent les calculs Monte-Carlo, pour une précision comparable.

\section{Conclusion et perspectives}

Le but de notre contribution n'est pas une présentation exhaustive de techniques possibles car elle dépasserait largement le volume prévu pour ce type d'information. Il nous a semblé opportun, au regard des résultats déjà obtenus, de mettre en évidence la faisabilité et l'intérêt de leur utilisation. La complexité de leur implémentation est toutefois un facteur important dont il faut tenir compte.

Les travaux effectués et déjà publiés dans ce domaine d'applications précis sont à ce jour extrêmement limités. Quelques essais avec l'apprentissage uniquement expérimental, naturellement trop laborieux avec le nombre de mesures assez limité, ont été tentés à la fin des années 90 (Wu et Zhu, 2000). La première tentative de rassembler les techniques RNA avec l'apprentissage par le Monte Carlo, en vue de la dosimétrie médicale, est à notre connaissance la référence de notre équipe (Mathieu et al., 2005). Il est pour le moment donc prématuré de juger de l'intérêt de tel ou tel algorithme (Blanpain et al., 2007), mais il est par contre indispensable que d'autres équipes s'aventurent sur cette voie assez prometteuse. Les différentes techniques envisagées (RNA et RàPC, entre autres) peuvent être 
développées soit individuellement, soit de manière couplée, promettant ainsi une diminution des temps de calcul et l'utilisation de méthodes MC encore plus performantes. L'obtention d'un profil de dose dû aux photons à une seule profondeur (2D) par la méthode Monte-Carlo (code de type EGS) requiert plusieurs heures, voire plusieurs jours de calculs selon les moyens informatiques utilisés et la précision demandée. Le réseau de neurones artificiels, une fois l'apprentissage fait, donne une réponse quasi-immédiate, en 3D, tout en conservant une précision du même ordre que le calcul MC.

En outre, il est aussi à noter que la recherche de nouvelles architectures informatiques peut aussi fournir des solutions intéressantes en pratique. L'utilisation des GPUs (Graphic Processing Units) dans les calculs de physique en est une illustration intéressante. Notamment, les premiers essais d'utilisation de GPUs pour l'accélération de codes de MC (Jahnke et al., 2008) démontrent un gain de temps de calcul allant d'un facteur 10 à 30 (en comparaison avec l'utilisation d'un CPU de coût similaire).

L'étape d'apprentissage dans le cadre des RNA pourrait être ainsi également améliorée. Quant à la technique RàPC, elle pourra trouver très certainement sa place aussi bien pour complémenter une expertise que pour créer des fantômes personnalisés. Le domaine de la radiothérapie externe constitue un champ d'exploitation de ces techniques d'apprentissage particulièrement intéressant et adapté. Il peut nous fournir une quantité de données suffisante grâce aux nombreux cas quotidiennement traités. Tout plan de traitement effectué par le passé (accompagné des résultats d'examens diagnostics), ou tout nouveau cas, devient à son tour une source potentielle d'informations venant enrichir les réseaux d'apprentissage. Sur le plan des simulations ou des modèles purement physiques, la radiothérapie et la radioprotection nécessitent, en règle générale, un nombre de paramètres d'entrée assez important mais limité. Après avoir réuni toutes les caractéristiques pertinentes (tailles de patients ou de victimes, composition des tissus, énergies utilisées, données biomécaniques, emplacement des tumeurs cancéreuses...), les problèmes posés ne demandent nullement des extrapolations extravagantes et se prêtent à priori à l'utilisation des méthodes informatiques mentionnées.

Remerciements. Les auteurs tiennent à remercier pour le soutien à niveaux différents : le comité local de la Ligue contre le cancer, la CAPM communauté d'agglomération du pays de Montbéliard, la région Franche-Comté, la SFRP et le Cancéropôle Grand-Est.

\section{RÉFÉRENCES}

Bahi J., Contassot-Vivier S., Makovicka L., Martin E., Sauget M. (2006a) Neural network based algorithm for radiation dose evaluation in heterogeneous environments, Lecture Notes in Computer Science (LNCS Subserie Bioinformatics-Springer Ver.) 4132(II), 777-787. 
Bahi J., Contassot-Vivier S., Makovicka L., Martin E., Sauget M. (2006b) Neurad /Agence pour la Protection des Programmes. No: IDDN.FR.001.130035.000.S.P.2006.000.10000.

Blanpain B., Mercier D., Barthe J. (2007) Calcul par réseaux de neurones de la dose déposée en radiothérapie par un faisceau fin dans un volume hétérogène, Schedae 25(2), 151-159.

Bopp M., Henriet J., Makovicka L., Morello B., Broggio D. (2009) A new CBR-Based application for voxelised phantom creation: REEPH. In: SinFra'09 - IPAL Symposium, February 18-20, 2009, Fusionopolis, Singapore, pp. 107-115, http://ipal.i2r.a-star.edu.sg/SinFra09.

Buffard E., Gschwind R., Makovicka L., David C. (2006) Monte Carlo calculations of the impact of a hip prothesis on the dose distribution, Nucl. Instr. Meth. B 251(1), 9-18.

Gschwind R., Buffard E., Masset H., David C., Makovicka L. (2008) Dosimetric Influence of hip prosthesis during radiotherapeutic treatement, Cancer Radiothérapie 12(2), 102-109.

Jahnke L.K.R., Fleckenstein J., Clausen S., Hesser J., Lohr F., Wenz F. (2008) GPU-acceleration of GEANT4-based Monte Carlo Simulations for Radiotherapy. In: Proceedings of the American Society for Therapeutic Radiology and Oncology 50th Annual Meeting, 1 September, 2008, Vol. 72, Issue 1, Supplement 1, Page S628.

Kafrouni H., Husson F., Morow S., Franchisseur E., Benkebil M. (2008) L'utilisation de la méthode de Monte Carlo pour la planification du traitement du cancer par radiothérapie devient une réalité. In: La dosimétrie individuelle des travailleurs et des patients : Mise en œuvre et perspectives, Journées de la SFRP, www.sfrp.asso.fr, 27-28 mai, 2008, Paris.

Kolodner J. (1993) Case-Based Reasoning. Morgan Kaufmann Publishers.

Mathieu R., Contassot-Vivier S., Guillerminet C., Gschwind R., Makovicka L., Bahi J. (2003) Prospective de la planification des traitements radiothérapeutiques basée sur les réseaux de Neurones. In: Journées SFRP-SFPM-FIRAM «Codes de calcul en radioprotection, radiophysique et dosimétrie », Journées SFRP, www.sfrp.asso.fr, 2-3 octobre, 2003, Sochaux.

Mathieu R., Gschwind R., Martin E., Makovicka L., Contassot-Vivier S., Bahi J. (2004a) Use of the neural networks in external radiotherapy. In: "Current Topics in Monte Carlo Treatment Planning », Advanced Workshop, May 3-5, 2004, Montréal.

Mathieu R., Martin E., Gschwind R., Makovicka L., Contassot-Vivier S., Bahi J. (2004b) Utilisation des réseaux d'apprentissage neuronaux en radiothérapie externe. In: $43^{\text {es }}$ Journées scientifiques de la SFPM, Journées SFPM, www.sfpm.asso.fr, 2-4 juin, 2004, Montpellier.

Mathieu R., Martin E., Makovicka L., Gschwind R., Contassot-Vivier S., Bahi J. (2005) Calculations of dose distributions using a neural network model, Phys. Med. Biol. 50, 1019-1028.

Pitts W., Culloch W.S. (1943) A logical calculus of ideas immanent in nervous activity, Bull. Math. Biophys. 5, 115-133.

Sauget M., Contassot-Vivier S., Bahi J., Martin E., Makovicka L. (2005) Utilisation des réseaux de neurones pour la conception d'un code de calcul dosimétrique en radiothérapie externe. In: $22^{e s}$ Journées LARD, http//clrwww.in2p3.fr/lard, 20-21 octobre 2005, Montbéliard.

Sauget M. (2007) Thèse de doctorat, Université de Franche-Comté, n 98, ED-SPIM, « Parallèlisation de problèmes d'apprentissage par des réseaux neuronaux. Application en radiothérapie externe ».

Vasseur A., Sauget M., Martin E., Contassot-Vivier S., Makovicka L., Bahi J. (2006) Utilisation d'un code de calcul dosimétrique basé sur les Réseaux de Neurones Artificiels et la méthode de Monte Carlo. In: $45^{\text {es }}$ Journées Scientifiques de la SFPM, Journées SFPM, www.sfpm.asso.fr, 7-9 Juin, 2006, Lyon.

Vasseur A., Makovicka L., Martin E., Sauget M., Contassot-Vivier S., Bahi J. (2008) Dose calculations using artificial neural networks: a feasibility study for photon beams, Nucl. Instr. Meth. B 266,1085-1093.

Wu X., Zhu Y. (2000) A neural network regression model for relative dose computation, Phys. Med. Biol. 45, 913-922. 